\title{
Green synthesis of metal nanoparticles using plant phytochemicals and their potential applications \\ Shib Shankar Dash*
}

\author{
Department of Chemistry, Govt. General Degree College, Salboni, Paschim Medinipur, West Bengal, India \\ Email: shiba.chem@gmail.com
}

Received- November 6, 2016 | Accepted: April 28, 2017 | Published online - April 29, 2017

\begin{abstract}
Various plant extract rich in phytochemicals have been utilized for the one step synthesis of metal nanoparticles at room temperature. The phytochemicals especially polyphenolic compounds are highly efficient to reduce metal ions and also stabilize them in nanometric dimension. Interestingly, no other stabilizing agent is necessary for such synthesis. The metal nanoparticles especially gold and silver using plants (plant tissue, plant extracts and living plant) has gained much interest in recent years because of their applications in pharmaceuticals, optoelectronic devices, drug delivery into cell and catalysis etc. In this review, I have addressed the one step facile synthesis of metal nanoparticles using different plant extracts, various methods of characterization and applications of metal nanoparticles.
\end{abstract}

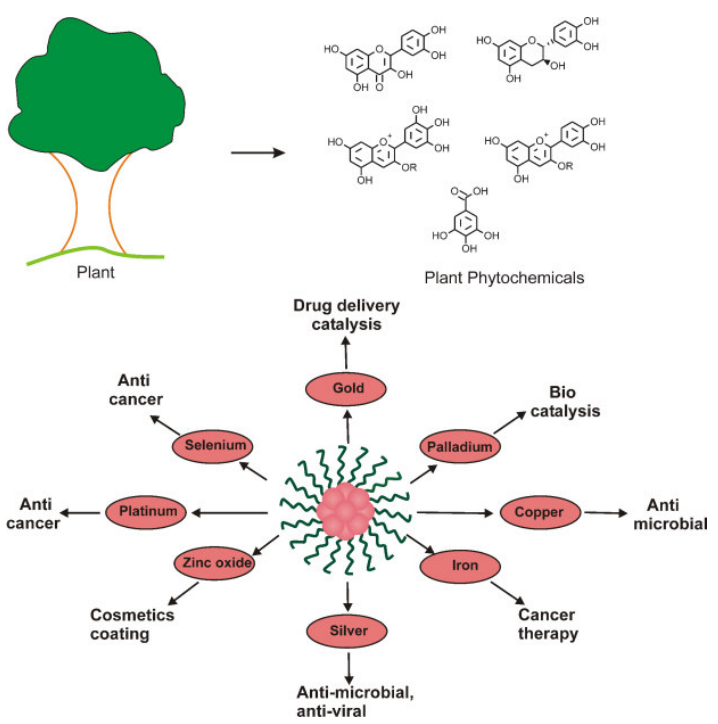

In Bengali: সাধারণ তাপমাত্রায় একধাপে ধাতব কণিকা তৈরীর জন্যে রাসায়নিক পদার্থে সমৃদ্ধ বিভিন্ন উদ্ভিদজাত পদার্থ ব্যাবহার করা যেতে পারে। রাসায়নিক পদার্থ বিশেযত পলিফেনোলিক যযাগগুলি ধাতব আয়ন বিজারন এবং তাদের ন্যানো পারিধিতে স্থায়ীত্ব দিতে খুবই সক্ষম। এটা আরোও আকর্ষণীয় কারন এই সংশ্লেযন পদ্ধতিতে অন্যকোন স্থিতকারী প্রতিনিধির প্রয়োজন হয় না। সাম্প্রতিক বছর গু লিতে উদ্ভিদজাত সংশ্লেষন দ্বারা প্রস্তুত ধাতব কণিকা, বিশেষত স্বর্ণ ও রৌপ্য কণিকা আরো বেশি আকর্যনীয় হয়েছে কারন ঔষধ, অপ্টোইলেকট্রনিক যন্ত্রাতি, ঔষধকোযে পাঠানো এবং অনুঘটক তৈরীতে বিশেষ ব্যাবহার রয়েছে। এই পর্যালোচনাটিতে, আমি উদ্ভিদজাত সংশ্লেযন ব্যাবহার করে এক ধাপে ধাতব কণিকা তৈরীর পদ্ধতি, বিভিন্ন পদ্ধতিতে তাদের বিল্লেষন এবং তাদের ব্যাবহার বর্ণনা করেছি।

Keywords: Plant phytochemicals, green synthesis, metal nanoparticles, gold nanoparticles, characterization, application.

\section{Introduction}

The goal of controlling materials on the nanoscale with the efficiency and elegancy of nature from a bottom up approach is a key ambition of nanoscience and nanotechnology. 1,2
Nanoparticles of different materials in a wide variety of shapes and sizes are a popular starting point due to their novel physical and chemical properties. $^{3}$ Metal nanoparticles of size range 1$100 \mathrm{~nm}$ have gained tremendous research interest in recent years due to their optoelectronic and magnetic properties which make them attractive for their diverse applications in 


\section{Prayogik Rasayan}

nanobiodiagnostics, ${ }^{4}$ pharmaceuticals, ${ }^{5}$ drug delivery, ${ }^{6}$ optoelectronic devices, ${ }^{7}$ non linear optical devices, chemical sensors, wireless electronic logic, memory schemes, ${ }^{8}$ catalysis, etc. ${ }^{9}$ Metallic nanoparticle shows completely different properties compare to bulk due to surface confinement of electrons. For example, the surface plasmon resonance (SPR) of these nanoparticles is widely studied due to its ease of characterization and coupling with electronic and magnetic properties. Electronic absorptions of nanoparticles can be tunable due to size and morphology. Through careful control of experimental parameters, different shapes of nanoparticle can be produced. In addition to the synthesis, the two dimensional and three dimensional assemblies of these materials becomes interesting due to fascinating properties in the field of nanoscience and nanotechnology. The design and synthesis of materials with potential applications can be achieved by controlling their size and shape at nanometer scale. Gold and silver nanoparticles are the most common ones used for biomedical applications and in the field of nanobiotechnology. Gold nanoparticles have been utilized in immunoassay, cancer nanotechnology (especially detection of cancer cells), etc. Gold nanoparticles are used as markers for biological screening test. After cellular uptake, they can act as precise and powerful heaters (thermal scalpels) to kill cancer. Moreover, gold nanoparticles are capable of inducing apoptosis in B cell-chronic lymphocytic leukemia (chronic lymphoid leukemia). Silver nanoparticles have drawn the attention of researchers in recent years because of their extensive applications in areas such as sensors, bio labeling, filters, antimicrobial deodorant fibers, cell electrodes, and antimicrobials. Antimicrobial properties of silver nanoparticles caused the use of these nano metals in different fields of medicine, various industries, packaging, accessories, cosmetics, health etc. Silver nanoparticles show potential antimicrobial efficacy against Gram-positive bacteria such as Bacillus subtilis, Staphylococcus aureus and Gram-negative bacteria such as Escherichia coli, Klebsiella aerogenes, and Klebsiella pneumonia, etc. ${ }^{10,11}$

\section{Green synthesis of metal nanoparticles}

The methods for the synthesis of gold nanoparticles mainly classified into two categories, one is "top down" approach and other is "bottom up" approach. ${ }^{12,13}$ In the top down synthetic approach, nanoparticles are prepared starting from suitable materials.

Figure 1: Top down and bottom up approach for the synthesis of metal nanoparticles.

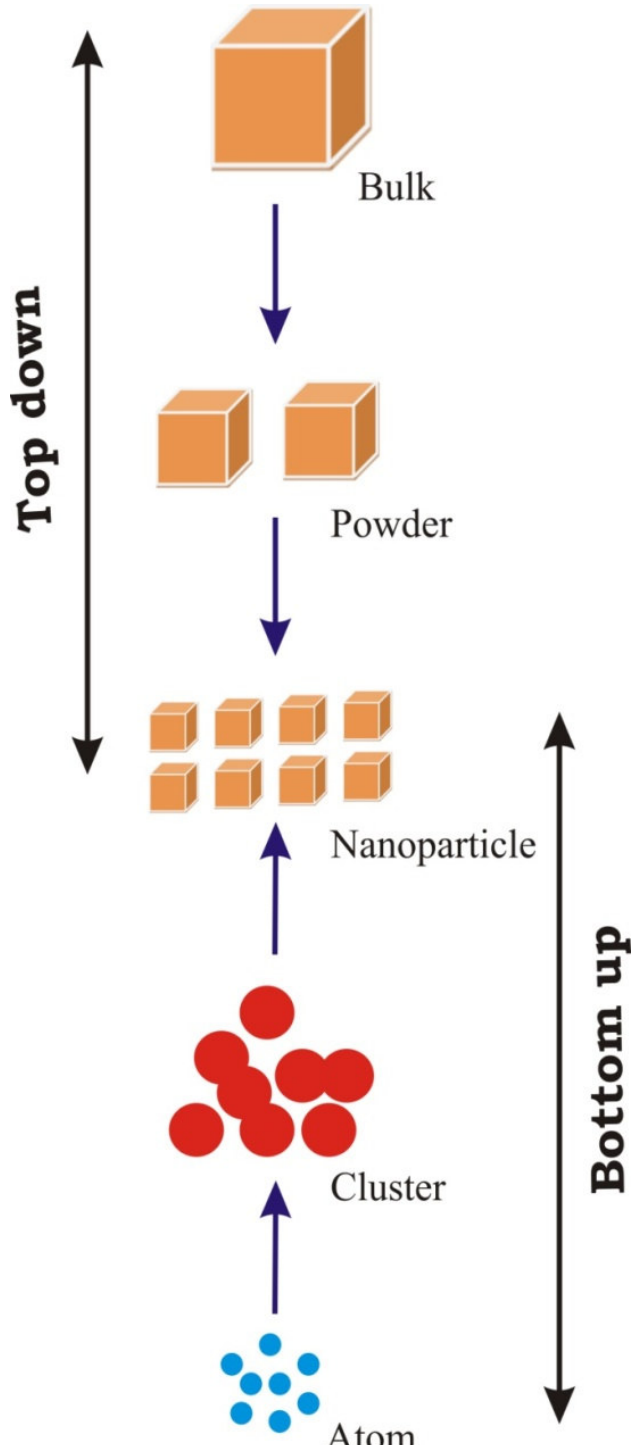

Atom 


\begin{tabular}{|c|c|c|c|c|}
\hline Plant source & Nanoparticle & Size $(\mathbf{n m})$ & Morphology & References \\
\hline Aloe vera & $\mathrm{Au}, \mathrm{Ag}$ & $50-350$ & spherical, triangular & 14 \\
\hline Azadirachta indica (neem) & $\begin{array}{l}\mathrm{Ag} / \mathrm{Au} \\
\text { bimetallic }\end{array}$ & $50-100$ & $\begin{array}{l}\text { spherical, triangular, } \\
\text { hexagonal }\end{array}$ & 15 \\
\hline Black tea leaf extracts & $\mathrm{Au}, \mathrm{Ag}$ & 20 & spherical, prism & 16 \\
\hline Ficus carica (Dumur) & $\mathrm{Au}$ & $13-14$ & trigonal, tetragonal, rod-like & 17 \\
\hline
\end{tabular}

Table 1: Biosynthesis of nanoparticles using plant extracts.

The reduction of size can be achieved by various chemical and physical methods such as mechanical milling, chemical etching, laser ablation, sputtering, etc. In top down synthetic approach the surface structure of the nanomaterials are destroyed. Therefore, this is the major limitation of this method because the surface chemistry and other physical properties of the nanoparticles are highly dependent on the surface structure. In bottom up synthesis nanoparticles are synthesized starting from smaller entities by joining atoms, molecules and smaller particles (Figure 1). The bottom up synthesis mostly relies on chemical and biological methods of production. In the earlier days, nanoparticles have been synthesized physically and chemically for a long time. But recently microorganisms and biological systems mediated synthesis of metal nanoparticles have been reported. The use of organisms in the synthesis of nanoparticles is rapidly developing due to their growing success and ease of formation of nanoparticles. Additionally, biosynthesis of metal nanoparticles is an environmentally friendly method (green chemistry) without use of harsh, toxic and expensive chemicals. Biosynthesis of metal nanoparticles using plant derived chemicals is currently under exploitation. Among various metal nanoparticles, the synthesis of gold and silver nanoparticles using plants (plant tissue, plant extracts and living plant) has gained renewed interest in recent years as a suitable alternative to chemical procedures and physical methods. As most of the plant extracts are rich source of phytochemicals including polyphenolic compounds, they may act both as reducing and capping agents in the synthesis and stabilization of nanoparticles, where no additional capping agent is necessary (Figure 2). Synthesis of metal nanoparticles using plant extracts is very cost effective, and therefore can be used as an economic and valuable alternative for the largescale production of metal nanoparticles.

\subsection{Green synthesis of nanoparticles using} plant extract: In recent years the synthesis of metal nanoparticles using various plant parts extract and suitable metal salt has drawn considerable attention to the scientific community as the method adopted in the green synthesis is simple, cost effective, and environmentally benign to nature (Table 1). The size and shape of the nanoparticles synthesized by the above methods was depended on the concentration of the plant extract, $\mathrm{pH}$, reaction time, temperature, etc. Chandran et al. reported the synthesis of gold and silver nanoparticles using the Aloe vera extract. ${ }^{14}$ Analysis of HRTEM images of the nanoparticles revealed that the nanoparticles are mostly spherical and triangular in shape with an average diameter of 50-350 nm. Gold and silver bimetallic nanoparticle of average diameter $50-100 \mathrm{~nm}$ can be prepared utilizing Azadirachta indica (neem) ${ }^{15}$ extract at room temperature. Electron microscopy revealed the formation of poly shaped nanoparticles. In another study, black tea leaf ${ }^{18}$ extracts were used in the synthesis of $\mathrm{Au}$ and $\mathrm{Ag}$ nanoparticles. The nanoparticles are stable and have different shapes, such as spheres, trapezoids, prisms and rods. The study has demonstrated that polyphenols and flavonoids were responsible for the synthesis of nanoparticles. 
2.2 Mechanism of synthesis of metal nanoparticles and its stabilization: Plants are the rich source of phenolic compounds. A schematic representation for the formation of AuNPs and their stabilization was given in Figure 4. Phenolic compounds and other easily oxidizable phytochemicals can reduce $\mathrm{Au}$ (III) to $\mathrm{Au}$ (0). The electron rich o-dihydroxy compounds present in the plant extract can easily form a five-membered chelated ring with the $\mathrm{Au}$ (III) ions. The redox reaction can take place in the chelated complex where the o-dihydroxy compounds can be oxidized to corresponding quinones with concomitant reduction of $\mathrm{Au}$ (III) ions to $\mathrm{Au}(0)$. Collision of the neighboring $\mathrm{Au}$ (0) atoms with each other may lead to the formation of the nano-sized gold particles. The AuNPs thus formed can be stabilized by the quinones, phenolic compounds, as well as other coordinating phytochemicals present in the plant extract. Further aggregation of the AuNPs is prevented by the stabilizing ligands surrounding the AuNPs.
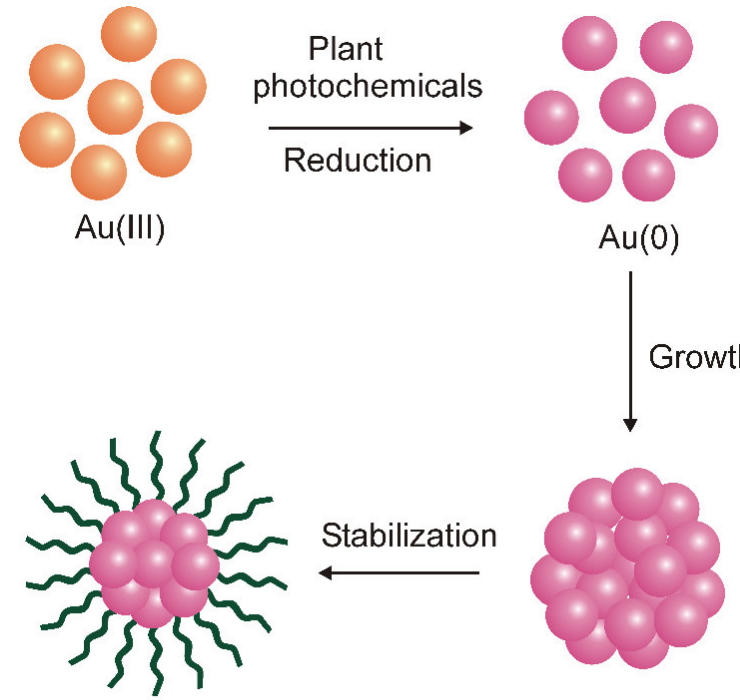

Stabilized AuNPs

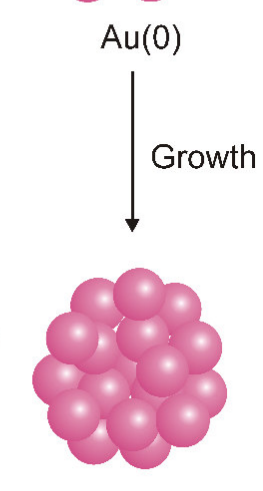

Assembly of $\mathrm{Au}(0)$
Figure 2: Synthesis and stabilization of metal nanoparticles by plant extracts.

\subsection{Plant mediated size controlled synthesis of} AuNPs: Synthesis of AuNPs of controlled size has drawn tremendous research interest in recent years because the nanoparticles of desired size have tremendous potential applications in different fields of science and technology. Recently, we have been published size controlled synthesis of AuNPs by fine tuning of the concentration of different plant extract. The average sizes of AuNPs synthesized with Saraca indica bark extract was 23-15 $\mathrm{nm}$ when concentration of bark extract increased from 80$200 \mathrm{mgL}^{-1}$ (Figure 4). A blue shift in the SPR band of the UV-visible spectroscopy also supported the phenomenon (Figure 3). ${ }^{19}$ AuNPs of controlled size can also be prepared by utilizing the juice extract of Punica granatum. ${ }^{20}$ In this study AuNPs of $36-23 \mathrm{~nm}$ size can be synthesized using Punica granatum juice extract of concentration 1400-2400 $\mathrm{mgL}^{-1}$.

\section{Characterization}

Nanoparticles were characterized according to their size, shape, surface area and dispertivity in solution. There are various techniques to characterize the nanoparticles such as: UVvisible spectrophotometry, transmission electron microscope (TEM), scanning electron microscope (SEM), atomic force microscopy (AFM), fourier transform infrared spectroscopy (FTIR), x-ray diffraction (XRD), selected area electron diffraction (SAED), energy disperse $\mathrm{X}$ ray spectroscopy (EDX) and dynamic light scattering (DLS) etc.

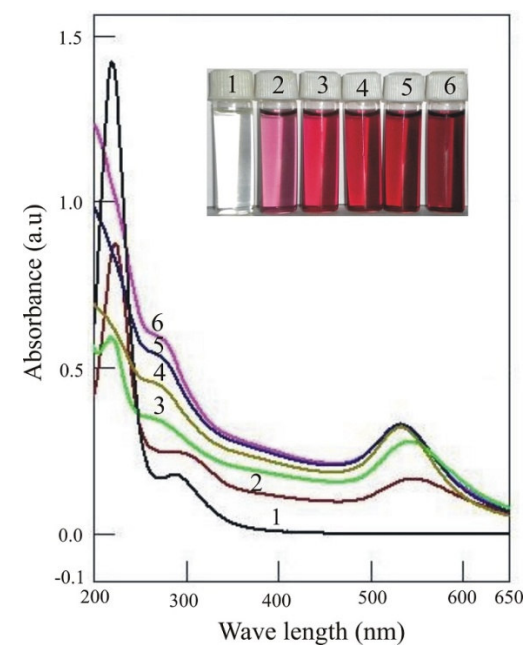

Figure 3: UV-visible spectra of (1) $\mathrm{HAuCl}_{4}(0.42$ $\mathrm{mM}),(2-6)$ AuNP's at 20, 40, 60, 80, $100 \mathrm{mgL}^{-1}$ concentrations of Saraca indica bark extract respectively. Inset: Photograph of the vials containing the solutions (after $24 \mathrm{~h}$ of mixing). Figure 3 was reproduced with permission from reference 19 . 
3.1 UV-visible spectroscopy: The UV-visible spectroscopy is a commonly and largely used technique to examine the formation of metal nanoparticles. Generally, when the photon energy (hv) is equal to the energy gap of
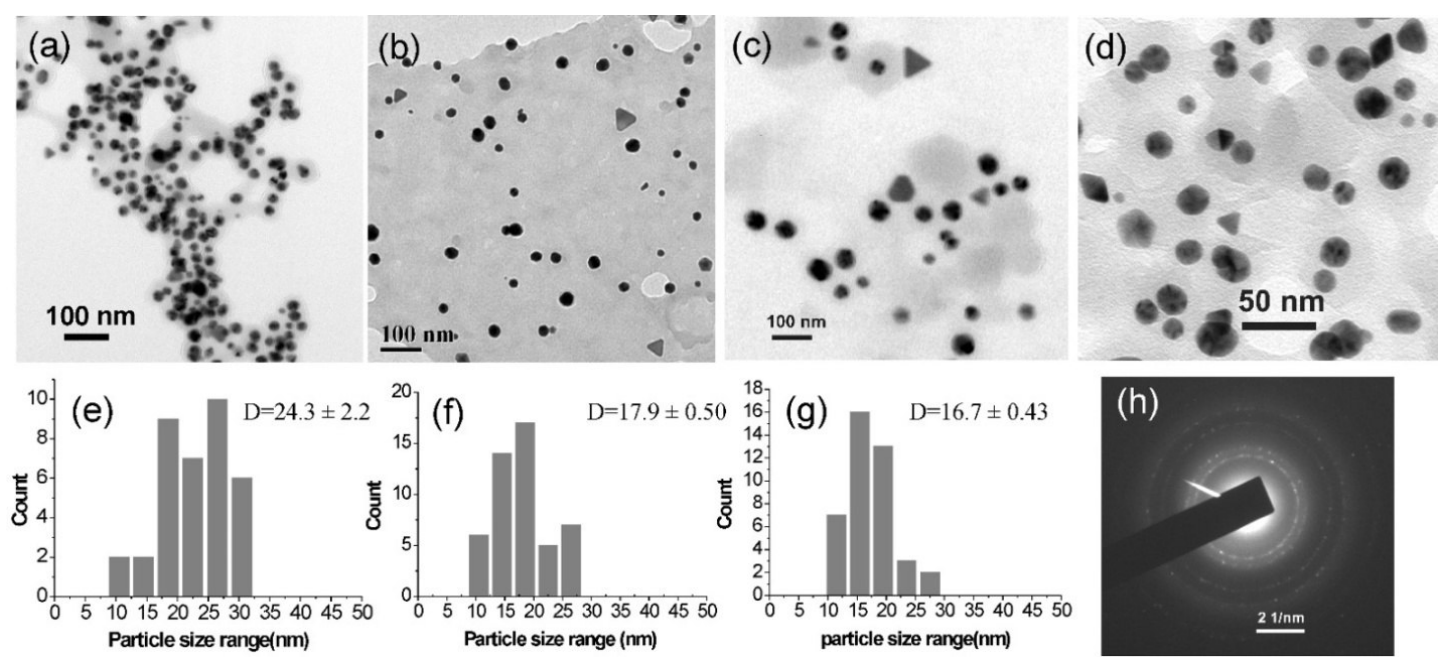

Figure 4: (a,b,c,d) TEM Images of AuNPs obtained with Saraca indica bark extract at 80, 100, 120 and $200 \mathrm{mgL}^{-1}$ concentration respectively, (e,f,g) Histograms are for the AuNPs at 100, 120 and $200 \mathrm{mgL}^{-1}$ of the bark extract. $\mathrm{D}=$ mean particle diameter, (h) SAED image of AuNPs. Figure 4 was reproduced with permission from reference 19.

semiconductor nanoparticles, electron moves from valence band to conduction band. If the size distribution of nanoparticles is narrow, the peak is very sharp. But if the size distribution is wide as all the particles contribute in absorption spectrum, which gives very broad peak. The characteristic Surface Plasmon Resonance (SPR) band in their respective region confirmed the formation of metal nanoparticles. The SPR band for most of the metal nanoparticles appeared in the range between $300-800 \mathrm{~nm}$, when the size of the metal nanoparticles falls in between 2-100 nm. For silver nanoparticles the SPR band appeared in the wave length range $400-450 \mathrm{~nm}$ and for gold nanoparticles it is $500-580 \mathrm{~nm}$.

\subsection{HRTEM, SEM, SAED and EDX studies:} It is another commonly used technique to investigate the shape of the nanoparticles. Scanning electron microscopy (SEM) and transmission electron microscopy (TEM) are used extensively for the study of morphology of the nanoparticles. TEM is a microscopic technique where a beam of electrons is transmitted through an ultra thin specimen, interacting with the specimen as they pass through. When electrons are incident on any specimen, they may back scattered, generate secondary electrons, transmit them or get scattered. TEM coupled with selected area electron diffraction (SAED) can provide important information on the crystallographic directions in the structures, helpful to understand the growth kinetics. It offers a unique capability to determine the crystal structure of individual nanomaterials such as nanocrystals, nanorods and crystal structures of different parts of sample. EDX analysis is one of the versatile techniques for determining the chemical composition of unknown sample. An EDX spectrum displays peaks corresponding to these energy levels for which the most X-rays has been received.

3.3 AFM studies: Atomic force microscopy (AFM) is also another useful tool to observe the three dimensional (3D) view of metal nanoparticles.

3.4 FTIR studies: FTIR spectroscopy is used to investigate the role of organic compounds in the reduction of metal ion into atomic state and 


\section{Prayogik Rasayan}

the stabilization of metal nanoparticles. The capping monolayer surrounding the nanoparticles and its nature, i.e. orientation, packing and density are generally characterized by FTIR spectroscopy.

3.5 X-ray diffraction study: $\mathrm{XRD}$ is used to know the crystal structure of the nanoparticles.

\section{Morphology of gold nanoparticle}

The investigations of morphologies of AuNPs have increased during the last decade, most often relying on the development of seed mediated synthetic methods. In this respect, AuNPs are probably the class of nanoparticles that has provided the largest variety of shapes and has been studied the most. AuNPs with the morphology of the five platonic solids-the tetrahedron (four triangles) hexahedron (cube, six squares), ${ }^{21}$ octahedron (eight triangles), dodecahedron (twelve pentagons), and icosahedrons (twenty triangles) ${ }^{22}$ and their aqueous synthesis is based on seed growth, reported by the Murphy research group. ${ }^{23}$ Now a day, the most extensively investigated onedimensional AuNPs is nanorods, nanowires, nanotubes and nanobelts, etc. Seed mediated growth lead to the formation of twinned gold nanorods (AuNRs). AuNRs with very high aspect ratio are known as $\mathrm{Au}$ nanowires. $\mathrm{Au}$ nanotubes (AuNTs) can be prepared by electroless deposition onto the pore walls of porous polymer membranes, as shown by the seminal study by Wirtz and Martin. ${ }^{24}$ Han and co-workers synthesized single-crystalline gold nanobelts (AuNBs) by using a combination of ultrasound irradiation and a d-glucose as a directing agent under ambient conditions. ${ }^{25}$ Two-Dimensional AuNPs i,e gold nanoplates are prepared through polymer template method, where the polymers acted as stabilizers, templates, and reductants no other extra stabilizing agent is necessary. Radhakrishnan and co-workers generated polygonal gold nanoplates in situ in a poly (vinyl alcohol) matrix through thermal treatment. ${ }^{26}$ Nanodumbbells, nanopods, nanostars, nanodendrites, nanocages, nanoframes are the examples of three dimensional gold nanoparticles. ${ }^{27}$

\section{Optical Properties of Anisotropic AuNPs: SPR and SERS}

Surface plasmon resonance (SPR) is the collective oscillation of electrons in a solid or liquid stimulated by incident radiation of light. The resonance condition is achieved when the frequency of light photons matches with the natural frequency of surface electrons oscillating against the restoring force of positive nuclei. The position of SPR peak largely depends upon the size, shape and structure of AuNPs. AuNRs (also the AuNDs and AuNTs) exhibit two plasmon bands: a strong longitudinal band in the near-infrared region and a weak transverse band, in the visible region which is very much similar to that of spherical AuNPs. Gold nanostars show a plasmon band of the core and multiple plasmon bands corresponding to the tips and core-tip interactions. ${ }^{28}$ Surface Enhanced Raman Spectroscopy or Surface Enhanced Raman Scattering (SERS) is a surface sensitive technique that enhances Raman scattering by molecules adsorbed on rough metal surfaces or by nanostructures such as plasmonic-magnetic silica nanotubes.

\section{Toxicology of AuNPs}

In general AuNPs are non toxic for cells. In contrast other nanoparticles such as carbon nanotubes, asbestos and metal oxides have drastic effect on cells. Anisotropic AuNPs have an enormous potential in theranostics. The in vivo toxicity of these AuNPs needs to be established before the delivery of drug into the cells. Therefore, in vitro and in vivo toxicology studies of AuNPs are necessary before use of a drug. In most of the cases spherical AuNPs are used for the study but only a few cases AuNRs have been used for biomedical applications. ${ }^{29}$ 


\section{Prayogik Rasayan}

\section{Application of nanoparticles}

Now a day, nanoparticle synthesized by various methods have been used extensively in various fields such as sensing, drug delivery, gene therapy, imaging of cancer cells, degradation of pesticides, antimicrobial agent and catalysis etc. Figure 5 shows the general applications of metal nanoparticles. Distinct physical and chemical properties of AuNPs possess them excellent scaffolds for their use in sensors. Mercury is a well known environmental pollutant released from various sources such as power plants, burning fossil fuels, etc. AuNPs are capable for sensing mercury in tap water samples at the parts-per-trillion (ppt) level. The

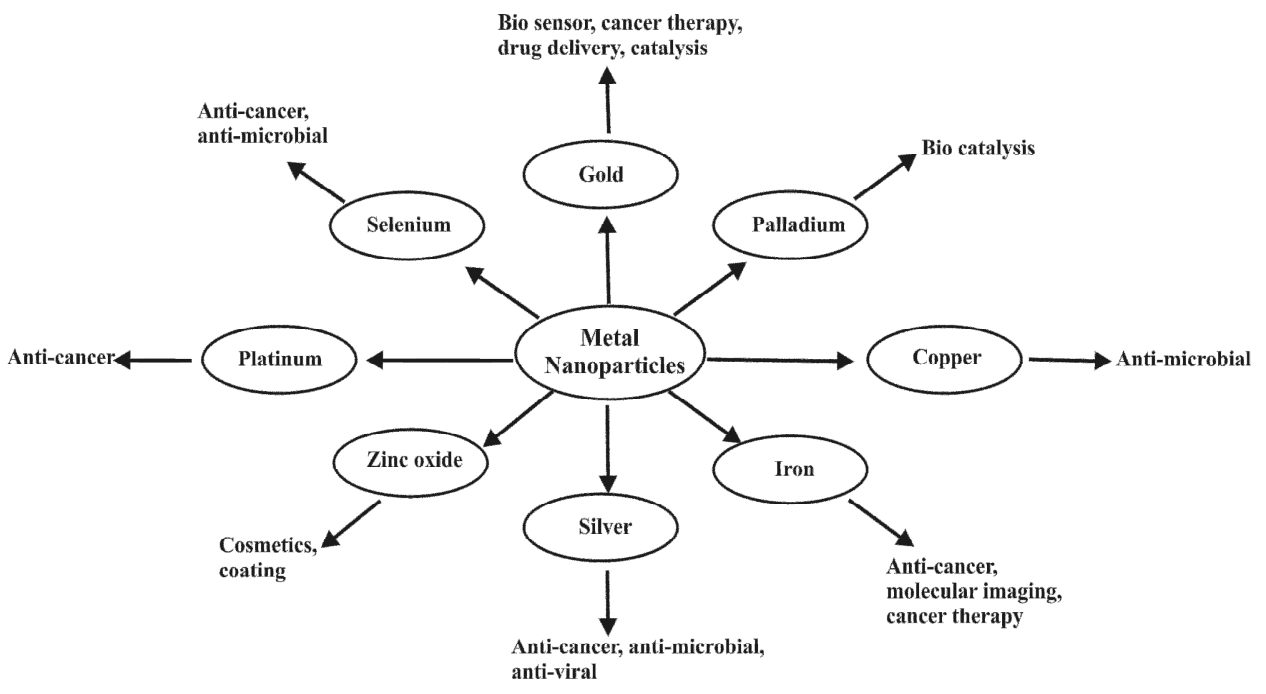

Figure 5: Metal nanoparticles and their various potential applications.

contamination of drinking water with arsenic poses a risk to whole world. Ray et al. reported the use of AuNPs in the selective detection of arsenic in groundwater using simple colorimetric and ultrasensitive dynamic light scattering assay. The unique optical, chemical, and biological properties of AuNPs make them attractive candidate for their applications in drug and gene delivery. Kim et al. observed that gold nanoparticles functionalized with oligonucleotides are able to activate immunerelated genes and pathways in human blood cells. $^{30}$ Both gold and silver nanoparticles have been commonly found to have broad spectrum of antimicrobial activity against human and animal pathogens. ${ }^{31,32}$ Gold is very popular for being chemically inert. The field of nanocatalysis has undergone an exponential growth during the past decades. ${ }^{33,34}$ Metallic nanoparticles in the nanometer size range provide very high surface to volume ratio which make them attractive for their application in catalysis. Recently, gold nanoparticles are largely used as catalyst for the sodium borohydride reduction of 4-nitrophenol to 4-aminophenol at room temperature. ${ }^{35}$ In a study, $0.2 \mathrm{~mL}$ of 4-nitrophenol $(0.05 \mathrm{mM}), 3.6$ $\mathrm{mL} \mathrm{NaBH}_{4}(15 \mathrm{mM})$ and $0.2 \mathrm{~mL}$ of distilled water were mixed together and UV-visible spectra was taken. The absorption peak at 318.5 $\mathrm{nm}$ for 4-nitrophenol shifted to $400 \mathrm{~nm}(\mathrm{~A}=$ 0.949) due to the formation of 4-nitrophenolate ion. Even though the reduction of 4-nitrophenol to 4-aminoaphenol is a thermodynamically favorable reaction $\left(\mathrm{E}^{0}\right.$ for 4-nitrophenol/4aminophenol -0.76 and for $\mathrm{H}_{3} \mathrm{BO}_{3} / \mathrm{BH}_{4}^{-}-1.33 \mathrm{~V}$ ), no reduction took place on standing the reaction mixture for several days due to a large kinetic barrier for the reduction reaction. Interestingly, in the presence of Saraca indica bark stabilized AuNPs $\left(0.2 \mathrm{~mL}, 60 \mathrm{mgL}^{-1}\right)$ the intensity of the absorption peak decreased immediately with a concomitant formation of a new peak at $295 \mathrm{~nm}$ 
region indicating the reduction of 4-nitrophenol to 4-aminophenol. The progress of the reaction was monitored by the UV-visible spectroscopy at different time intervals (Fig. 6). The absorption peak of 4-nitrophenolate ion (at $400 \mathrm{~nm}$ ) completely disappeared and the solution became almost colorless indicating the completion of reduction reaction. The UV-visible data at different time interval have been utilized for the

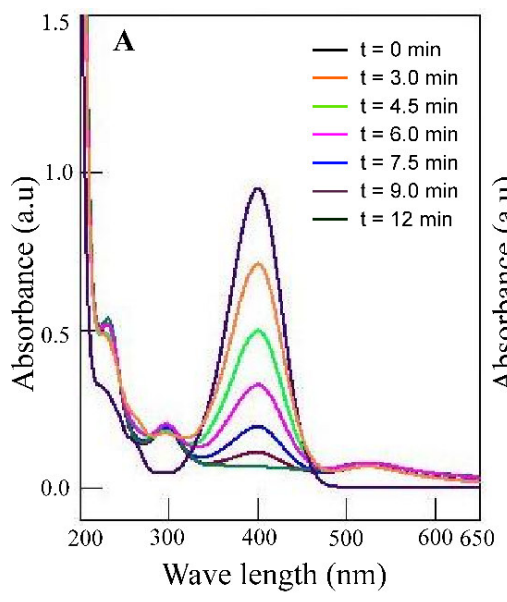

determination of catalytic rate constant (k). Since the concentration of $\mathrm{NaBH}_{4}$ greatly exceeds the concentration of 4-nitrophenol the reaction rate can be assumed to be pseudo first order. From the plot of $\ln \mathrm{A}$ vs time, the rate constant for the reduction reaction was calculated to be $0.25 \mathrm{~min}^{-}$ ${ }^{1}$. In the presence of $0.3 \mathrm{~mL}$ of the stabilized AuNP solution $\left(60 \mathrm{mgL}^{-1}\right)$ the rate constant was calculated to be $0.29 \mathrm{~min}^{-1}$.

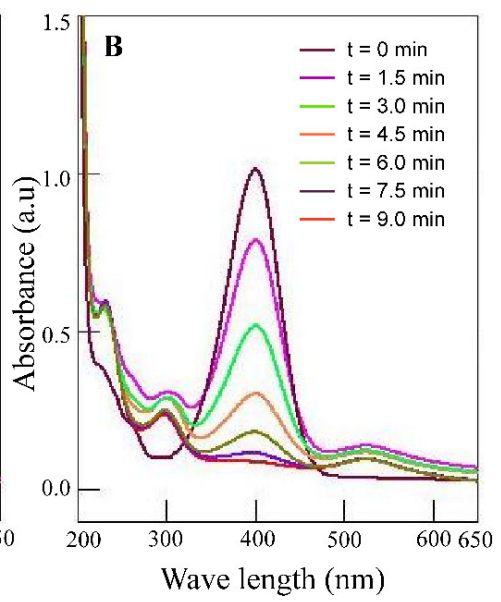

Fig. 6: UV-visible Spectra at different time interval during catalytic reduction of 4-nitrophenol to 4aminophenol; (A) using $0.2 \mathrm{ml}$ and (B) $0.3 \mathrm{ml}$ of stabilized AuNPs. Figure 6 was reproduced with permission from reference 19 .

\section{Conclusion}

Synthesis of metal nanoparticles using various plant phytochemicals are attractive because the method adopted here is simple, cost effective, time saving, environmentally benign and easily scaled up. Various classes of biomolecules present in plants such as proteins or enzymes, amino acids, polysaccharides, alkaloids, flavanoids, tannins, polyphenols, hydroxy acids and vitamins plays chief roles for the reduction of $\mathrm{Au}$ (III) to $\mathrm{Au}(0)$ atoms and their stabilization in nanometric dimension. The plant extract mediated synthesis can offer nanoparticles of a controlled size and morphology. Metal

nanoparticles are attracting because of their diversified applications in catalysis, cancer cell imaging, drug delivery sensors, anti-microbial activity, cosmetics etc. The easy production of metal nanoparticles using plant extracts and their potential applications in the diversified areas will be useful in the advancement of nanoscience and nanobiotechnology. Apart from the artistic appeal, bio modulation will be of tremendous interest to address the environmental concerns associated with the toxic chemicals used in the chemical method.

\section{Acknowledgement}

I thank Professor Braja Gopal Bag for his encouragements. I thank my all labmates for the continuous support. I also thank to Vidyasagar University for giving me an opportunity to fulfill my research by offering me all kind of instrumental assistance. CSIR-New Delhi is highly acknowledged for the finalcial support. 


\section{Author Information}

Dr. Shib Shankar Dash received his B.Sc. in Chemistry from Midnapore College, Vidyasagar University, India in 2007 and M.Sc. in Chemistry from

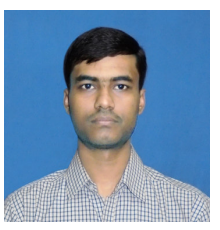
Vidyasagar University, India in 2009. He is completed his $\mathrm{PhD}$ in the year 2015 from the Department of Chemistry and Chemical Technology, Vidyasagar University, Midnapore, India under the supervision of Professor Braja Gopal Bag. Currently, he has joined as Assistant Professor at the department of Chemistry, Govt. General Degree College, Salboni, Paschim Medinipur. His research interest involves the utilization of plant based renewable chemicals in Supramolecular Chemistry and nanoscience.

\section{References}

1. P. S. Christopher, G. F. David, L. Raphael, J. Mater. Chem., 2011, 21, 12181.

2. V. Biju, Chem. Soc. Rev., 2014, 43, 744.

3. P. C. Ray, Chem. Rev. 2010, 110, 5332.

4. J. Gong, C. B. Mullins, Acc. Chem. Res., 2009, 42, 1063.

5. Y. Zhang, X. Cui, F. Shi, Y. Deng, Chem. Rev., 2012, 112, 2467.

6. K.G. Thomas, P. V. Kamat, Acc. Chem. Res., 2003, 36, 888 .

7. P. Laura, V. Alberto, Acc. Chem. Res., 2014, $47,855$.

8. J. Xie, C. Pan, A. Abdukader, C. Zhu, Chem. Soc. Rev., 2014,43, 5245.

9. S. K. Ghosh, T. S. Pal, Chem. Rev., 2007,107, 4797.

10. D. M. Ali, N. Thajuddin, K. Jeganathan, M. Gunasekaran. Colloids Surf B Biointerfaces, 2011, 85, 360 .

11. K. Amarnath, J. Kumar, T. Reddy, V. Mahesh, S. R. Ayyappan, J. Nellore, Colloids Surf B Biointerfaces, 2012, 94, 254.

12. S. Iravani, Green Chemistry, 2011, 13, 2638.

13. A. K. Mittal, Y. Chisti, U. C. Banerjee, Biotechnology Advances, 2013, 31, 31346.

14. S. P. Chandran, M. Chaudhary, R. Pasricha, A. Ahmad, M. Sastry, Biotechnol Prog, 2006, 22, 577-83.
15. S. S. Shankar, A. Rai, A. Ahmad, M. Sastry, J Colloid Interface Sci, 2004, 275,496-502.

16. N. A. Begum, S. Mondal, S. Basu, R. A. Laskar and D. Mandal, Colloids Surf., B, 2009, 71, 113-118.

17. S. S. Dash, B. G. Bag, A. Midya, Int. J. App. Chem., 2013, 1, 1-7.

18. N. A. Begum, S. Mondal, S. Basu, R. A. Laskar, D. Mandal, Colloids Surf. B, 2009, $71,113$.

19. S. S. Dash, R. Majumdar, A. K.Sikder, B. G. Bag, B. K. Patra, App Nanosci., 2014, 4, 485.

20. S. S.Dash, B. G.Bag, App Nanosci., 2014, 4, 55.

21. F. Kim, S. Connor, H. Song, T. Kuykendall, P. Yang, Angew. Chem. 2004, 116, 3759.

22. M. L. Personick, M. R. Langille, J. Zhang, N. Harris, G. C. Schatz, C. A. Mirkin, J. Am.Chem. Soc., 2011, 133, 6170.

23. T. K. Sau, C. J. Murphy, J. Am. Chem. Soc., 2004, 126, 8648 .

24. M. Wirtz, C. R. Martin, Adv. Mater., 2003, 15,455 .

25. J. Zhang, J. Du, B. Han, Z. Liu, T. Jiang, Z. Zhang, Angew. Chem., 2006, 118, 1134.

26. S. Porel, S. Singh, T. P. Radhakrishnan, Chem. Comm., 2005, 2387.

27. T. H. Lin, C. W. Lin, H. H. Liu, J. T. Sheu, W. H. Hung, Chem. Comm., 2011, 47, 2044.

28. E. Hao, R. C. Bailey, G. C. Schatz, J. T. Hupp, S. Li, Nano Lett., 2004, 4, 327.

29. E. Boisselier, D. Astruc, Chem. Soc. Rev., 2009, 38, 1759.

30. E. Kim, R. Schulz, P. Swantek, K. Kunstman, M. H. Malim, S. M. Wolinsky, Gene Therapy, 2012, 347.

31. A. Shome, S. Dutta, S. Maiti, P. K. Das, Soft Matter, 2011, 7, 3011.

32. S. Dutta, T. Kar, D. Mandal, P. K. Das, Langmuir, 2013, 29, 316.

33. G. Schmid, Chem. Rev., 1992, 92, 1709.

34. A. J. Hoffman, G. Mills, H. Yee, M. Hoffmann, J. Phys. Chem., 1992, 96, 5546.

35. S. S. Dash, B. G. Bag, App. Nanosci., 2014, 3, 55 . 